\title{
CRISPR Activation/Inhibition Experiments Reveal that Expression of Intronic MicroRNA miR-335 Depends on the Promoter Activity of its Host Gene Mest
}

Mathilde Courtes ${ }^{1}$, Céline Lemmers ${ }^{2}$, Anne Le Digarcher ${ }^{1}$, Ilda Coku ${ }^{1}$, Arnaud Monteil ${ }^{2}$, Charles Hong $^{3, \#}$, Annie Varrault ${ }^{1}$, and Tristan Bouschet ${ }^{1, *}$

${ }^{1}$ Institut de Génomique Fonctionnelle, Université de Montpellier, CNRS, INSERM, Montpellier, France 2Plateforme de Vectorologie de Montpellier (PVM), BioCampus Montpellier, Université de Montpellier, CNRS, INSERM, Montpellier, France

${ }^{3}$ Vanderbilt University School of Medicine Nashville, Nashville, USA

\#Present address: University of Maryland School of Medicine, Baltimore, MD, USA

*For correspondence: tristan.bouschet@igf.cnrs.fr

Keywords: microRNA; CRISPR; CRISPRa; CRISPRi; pluripotent stem cells; mouse; Mest; miR-335. 


\section{ABSTRACT}

MicroRNAs are small non-coding RNAs that act as rheostats to modulate gene expression during development, physiology, and disease. Approximately half of mammalian microRNAs are intronic. It is unknown whether intronic miRNA transcription depends on their host gene or a microRNA-specific promoter. Here, we show that CRISPR inhibition of host gene Mest downregulated hosted miR-335 in mouse embryonic stem cells and brain organoids. Reciprocally, CRISPR transactivation of Mest upregulated miR-335. By contrast, activation of miR-335 predicted promoter had no effect. Thus, intronic miR-335 expression depends on the promoter activity of its host gene. This approach could serve to map microRNA promoters. 


\section{INTRODUCTION}

microRNAs (miRNAs) are short non-coding RNAs that play a central role in regulating gene expression in plants and animals (Bartel 2018; Jones-Rhoades et al. 2006). miRNAs impact on development and physiology, and are dysregulated in diseases, including cancer (DeVeale et al. 2021; Schanen and Li 2011; Xue et al. 2021). Stringent gene annotations suggest that there are $~ 500$ miRNAs in mice (Chiang et al. 2010) and humans (Fromm et al. 2015). It is estimated that approximately half of mammalian miRNAs are intronic (Meunier et al. 2013; Rodriguez et al. 2004; Hinske et al. 2014).

miRNAs biogenesis sequentially involves transcription, cleavage of the miRNA hairpin precursor out of the primary transcript, transport of intermediate forms, and loading of the mature miRNA into the RNA-induced silencing complex (Bartel 2018; Westholm and Lai 2011; Ha and Kim 2014). The mechanisms that regulate miRNAs transcription, a key factor of miRNA abundance and tissue-specific expression, are not well defined, in particular for intronic miRNAs. Intronic miRNAs were first observed as frequently co-regulated with their host genes (Baskerville and Bartel 2005; He et al. 2012; Liang et al. 2007; Rodriguez et al. 2004; Seitz et al. 2004), suggesting that their transcription depends on the promoter activity of the host gene. By contrast, recent work suggests that most intronic miRNAs are not co-regulated with their host genes, which is supported by the fact that they have independent transcription start sites (Steiman-Shimony et al. 2018). Many additional studies have tried to map miRNA promoters using bioinformatics tools (Chen et al. 2019). For instance using chromatin modifications (Ozsolak et al., 2008) or deepCAGE data (Marsico et al., 2013) it was estimated that $\sim 30 \%$ of intronic miRNAs have independent promoters. To our knowledge, whether the transcription of an intronic miRNA depends on the promoter activity of the host gene or a miR-specific promoter has not been tested experimentally.

Mest (Mesoderm-specific transcript) is a protein-coding gene that hosts miR-335 in one of its introns. Mest and miR-335 are highly conserved during evolution and frequently co-regulated (Hiramuki 
et al. 2015; Liang et al. 2007; Ronchetti et al. 2008; Tomé et al. 2011; Yang et al. 2014). This suggests that Mest and miR-335 are controlled via common regulatory sequences, possibly Mest promoter. In addition, based on a luciferase assay, miR-335 was proposed to have an independent promoter located in a Mest intron (Zhu et al. 2014).

Here, to get insights into the mechanisms of transcription of an intronic miRNA, we have applied CRISPR/Cas9 -Clustered regularly interspaced short palindromic repeats (CRISPR) and CRISPRassociated protein 9 (Cas9)- technologies to Mest and intronic miR-335. More specifically, we have used CRISPR activation (CRISPRa) and CRISPR inhibition (CRISPRi) where a cleavage defective Cas9 (dCas9) is fused to either activators or repressors of transcription (Konermann et al. 2015; Yeo et al. 2018; Gilbert et al. 2013) and directed these complexes to the endogenous promoter sequences of Mest or to the predicted promoter of miR-335.

\section{RESULTS \& DISCUSSION}

\section{CRISPRi of host gene Mest suppresses the expression of hosted miR-335 in embryonic stem} cells

miR-335 is located in an intron of the protein-coding gene Mest (Fig. 1A, B) and is transcribed from the same DNA strand as its host gene, a common feature of intronic miRNAs (Hinske et al. 2014). Mest has one distal promoter (D) and one proximal promoter (P). Mest is highly expressed in mouse embryonic stem cells (mESCs) and Mest transcripts originate predominantly from the proximal promoter (P) (Fig. 1A, generated from previously published RNA-seq experiments (Bouschet et al. 2017)). Furthermore, miR-335-3p was reported to be expressed in mESCs (Kingston and Bartel 2019).

We reasoned that if miR-335 expression depends on the activity of Mest promoters, then repressing transcription at Mest promoters in mESCs with CRISPRi should decrease miR-335 transcripts. Using Hyper-piggyBac transposase (Yusa et al., 2011), we first generated a CRISPRi mESC line that stably expressed dCas9 fused to the repressors of transcription KRAB and MeCP2. dCas9-KRAB-MeCP2 was previously shown to efficiently repress a vast panel of genes in HEK293T 
cells (Yeo et al. 2018). CRISPRi mESCs (characterized in Supplemental Fig. S1) were transduced with lentiviruses that express either a control sgRNA (no match in the mouse genome) or a sgRNA targeting either the distal or the proximal promoter of Mest (Supplemental Fig. S2A). sgRNAs targeting Mest proximal promoter $\mathrm{P}$ downregulated Mest while targeting distal promoter $\mathrm{D}$ had no obvious effect (Supplemental Fig. S2B). Thus, as expected, CRISPRi was efficient only when targeting the active Mest promoter. Levels of the neighboring gene Copg2 were unaffected (Supplemental Fig. S2C).

We then selected two CRISPRi mESC clones expressing the control sgRNA and two clones expressing the sgRNA Mest P2 for further analyses (Fig. 1B). There was a >100 fold-downregulation of Mest in CRISPRi Mest clones compared to CRISPRi control clones (Fig. 1C). By contrast, Copg2 expression was unaffected (Fig. 1D). We next measured the levels of miR-335-3p and miR-335-5p, the final products of miR-335 biogenesis, by gene-specific RT followed by qPCR with Taqman probes. In CRISPRi Mest clones, miR-335-3p and miR-335-5p levels were reduced to less than $1 \%$ of levels measured in CRISPRi control clones (Fig. 1E, F), a massive downregulation that paralleled well that of Mest (Fig. 1C). Thus, the transcriptional activity of Mest proximal promoter is required for the expression of intronic miR-335 in mESCs.

\section{Mest promoter activity is required for $\mathbf{m i R}-335$ expression in brain organoids}

Next, we determined whether miR-335 expression dependency on Mest promoter persists upon differentiation of mESCs into brain organoids. Brain organoids were generated from mESCs according to a published protocol (Eiraku et al. 2008) with slight modifications (see Materials and Methods). RNAseq experiments on these brain organoids show enrichment in Gene Ontology Terms such as 'forebrain generation of neurons' after eight days of differentiation and 'telencephalon development' and 'action potential' after 15 days of differentiation (Bouschet and co-workers, unpublished).

As expected, brain organoids contained neural progenitors of dorsal identity (NESTIN+PAX6+ cells) after 8 days of differentiation, and neurons (TUBB3+ cells), including some neurons that expressed the cortical marker TBR1 after 15 days of differentiation (Fig. 2A). 
Mest and miR-335 transcripts were upregulated during the generation of brain organoids from CRISPRi mESCs expressing the control sgRNA (Fig. 2B). By contrast, Mest RNA was barely

77

remains repressed in differentiated cells. Importantly, miR-335 mature products were also barely detectable in these organoids (Fig. 2B). Thus, the activity of Mest promoter is required for miR-335 expression in both undifferentiated mESCs and their neural progeny.

\section{CRISPRa on Mest increases the expression of hosted miR-335}

We next tested whether transactivating Mest promoter is sufficient to increase miR-335 levels and therefore mirrors CRISPRi loss of function experiments. mESCs stably expressing the CRISPRa Synergistic Activation Mediator (SAM) module (Bonev et al. 2017) -composed of three transactivators (Konermann et al. 2015)- were transduced with lentiviruses expressing either a control sgRNA or a sgRNA targeting Mest (D) or (P) promoter (Supplemental Fig. S3A) -as described for CRISPRi-. Transactivating Mest distal promoter efficiently increased Mest transcripts (Supplemental Fig. S3B). By contrast, transactivating Mest proximal promoter with sgRNAs P1 and P2 had no major effect on Mest transcript level (Supplemental Fig. S3B), likely because this promoter is already very active in ESCs (Fig. 1A). The level of Copg2 was not altered by any of the three Mest sgRNAs (Supplemental Fig. S3C).

We selected for further analysis two CRISPRa control clones and two CRISPRa Mest clones (expressing the D sgRNA, Fig. 3A). On average, there was a 3.2 fold increase in Mest transcript in CRISPRa Mest clones compared to clones expressing the control sgRNA (Fig. 3B). As for CRISPRi, Copg2 expression was unaffected (Fig. 3C). Strikingly, the levels of both miR-335-3p and miR-335-5p also increased by a $\sim 3$-fold (Fig. 3D, E). Thus, activating the distal promoter of Mest with CRISPRa/SAM is sufficient to increase hosted miR-335 levels in mESCs. 
CRISPRa on miR-335 putative promoter does not affect $m i R-335$ levels

100

101

102

A previous study, based on luciferase assays performed in HEK293T cells, suggests that the sequence upstream of miR-335 (situated in a Mest intron) has some promoter activity (Zhu et al. 2014). Thus, we next tested whether we could upregulate miR-335 by directing SAM to this genomic region.

Because SAM efficiency correlates with baseline expression levels of the targeted gene - the fold of upregulation is inversely correlated with basal transcript level- (Konermann et al. 2015), and to maximize the chance to increase miR-335, SAM experiments were performed on cells with lower baseline levels of miR-335 than mESCs. We observed that miR-335-3p and miR-335-5p levels were respectively 13 and 47 times lower in MEFs compared to mESCs (Fig. 4A, B). Mest expression was also 60 times less expressed in MEFs than in mESCs (Fig. 4C), adding further support for the coregulation of Mest and miR-335.

We designed three sgRNAs $(\mu 1, \mu 2$, and $\mu 3)$ in the putative miR-335 promoter -a region named pro2 in (Zhu et al. 2014)- and compared their efficiency in upregulating miR-335 to sgRNAs that target Mest promoters (Fig. 4E). sgRNAs P1 and P2 (which target Mest P promoter) strongly upregulated Mest (Fig. 4E) but also miR-335 mature products in SAM MEFs (Fig. 4G, H). The upregulation of Mest was much higher in MEFs than in mESCs, as expected from their relative Mest baseline levels (see Fig. 4C). By contrast, the three sgRNAs that target the putative promoter of $m i R-335(\mu 1, \mu 2$, and $\mu 3)$ did not affect miR-335-3p nor miR-335-5p levels (Fig. 4G, H). Thus, this genomic sequence likely does not regulate miR-335 expression in MEFs. We cannot rule out that $m i R$-335 has an independent promoter located in another region. In this context, prediction of miR-335 promoter location using DeepCAGE data (Marsico et al. 2013) suggests that there could be several miR-335 promoters depending on the tissue. According to Marsico and coworkers, the most probable miR-335 promoters are Mest $(\mathrm{D})$ and $(\mathrm{P})$ promoters - what we confirmed experimentally here-, and less probably, a third region situated in another intron of Mest.

Data obtained in MEFs also revealed that transactivating Mest $(\mathrm{P})$ promoter resulted in a strong increase in Mest and miR-335 while transactivating (D) promoter had moderate effects. This contrasts 
with results obtained in ESCs where the most potent sgRNAs were those targeting the (D) promoter (Fig. 3 and Supplemental Fig. S3). Taken together, these data suggest that transcriptional activation of one or the other Mest promoter, depending on the cell type, is sufficient to increase the levels of intronic miR-335. This also supports the existence of primary transcripts, originating either at (D) or (P) promoters, that contain both Mest and miR-335 precursors.

To conclude, CRISPRa and CRISPRi experiments on Mest and miR-335 in mouse cells reveal that transcription of an intronic miRNA is regulated by the promoter of its host gene. Previous works propose that evolutionarily conserved intronic miRNAs, such as miR-335, are more frequently coexpressed with host genes than recently appeared intronic miRNAs (He et al. 2012; Steiman-Shimony et al. 2018). This suggests that the transcription of conserved intronic miRNAs depends on the host promoter while recently appeared intronic miRNAs tend to have independent promoters. To test these predictions our CRISPRa/i approach could be used to map miRNA promoters on a genome-wide scale.

\section{MATERIALS AND METHODS}

\section{Cell culture}

E14Tg2a mouse ESCs and their CRISPRa and CRISPRi derivatives were cultivated on gelatine coated dishes and maintained pluripotent in Serum/Lif media as described (Varrault et al. 2018). Organoids were generated in 96-well (U-bottom) Ultra-Low Attachment plates (Sumitomo) by seeding 3000 ESCs in corticogenesis medium 1: DMEM/F-12/GlutaMAX supplemented with $10 \% \mathrm{KSR}, 0.1 \mathrm{mM}$ of nonessential amino acids, $1 \mathrm{mM}$ of sodium pyruvate, $50 \mathrm{U} / \mathrm{ml}$ penicillin/streptomycin, $0.1 \mathrm{mM}$ of 2 mercaptoethanol (Sigma), $1 \mu \mathrm{M}$ DMH1-HCl (in house synthesized, Vanderbilt University) and $240 \mathrm{nM}$ IWP-2 (Tocris). On day 8 of differentiation, organoids were transferred to bacterial plates (Greiner) in corticogenesis medium 2: DMEM/F-12/GlutaMAX supplemented with N2 and B27 (without vitamin A) supplements, $500 \mu \mathrm{g} / \mathrm{ml}$ of BSA, $0.1 \mathrm{mM}$ of non-essential amino acids, $1 \mathrm{mM}$ of sodium pyruvate, 0.1 $\mathrm{mM}$ of 2-mercaptoethanol, and 50U/ml penicillin/streptomycin. Immortalized CRISPRa (SAM) MEFs (gift from Giacomo Cavalli's lab, unpublished) were cultivated in DMEM supplemented with 10\% FBS 
and $50 \mathrm{U} / \mathrm{ml}$ penicillin/streptomycin. All media components were from Life Technologies unless otherwise stated. Cell lines were routinely tested for the absence of mycoplasma (Mycoalert, Lonza).

\section{Generation of constructs expressing sgRNAs}

153 sgRNA sequences targeting Mest promoters were designed using CRISPick

154 https://portals.broadinstitute.org/gppx/crispick/public (formerly GPP sgRNA Design tool) or manually.

155 sgRNAs that target the putative miR-335 promoter (mm10_dna range=chr6_30740830-30741300) were 156 designed using CHOPCHOP (Labun et al. 2019). Pairs of oligonucleotides (Eurofins) were annealed 157 and subcloned into either sgRNA(MS2) cloning backbone (Addgene Plasmid \#61424) or Lenti 158 sgRNA(MS2)_zeo backbone (Konermann et al., 2015) (Addgene plasmid \# 61427) that were previously 159 digested with either Bbsl or BsmBI (NEB), respectively, and purified on a Chromaspin column 160 (Clontech). All constructs were verified by Sanger sequencing (Genewiz). sgRNA sequences are listed 161 in Supplemental Table S1.

\section{Lentiviruses production}

163 Lentiviruses were prepared as described elsewhere (Lin et al. 2002). Briefly, lentiviral transfer vectors were co-transfected with the HIV packaging plasmid psPAX2 and the plasmid pMD2G (coding for the vesicular stomatitis virus envelope glycoprotein G), in HEK-293T cells by the calcium phosphate method. Supernatants were collected at day 2 post-transfection and concentrated on sucrose by 167 ultracentrifugation at $95528 \mathrm{~g}$ for $1.5 \mathrm{~h}$ at $4^{\circ} \mathrm{C}$.

\section{Generation of CRISPRi ESC lines using PiggyBac Transposition}

169 E14Tg2a mouse ESCs were co-transfected with pCMV-HA-HyperpiggyBase (Yusa et al., 2011) and pB-dCas9-KRAB-MecP2 (Yeo et al. 2018) (Addgene plasmid \# 110824) using a Neon transfection

171 system (Life Technologies). Forty-eight hours post-transfection, cells were selected using Blastidicin

$172(15 \mu \mathrm{g} / \mathrm{ml}$, SIGMA). Stable pB-dCas9-KRAB-MeCP2 ESCs (CRISPRi ESCs) were then transduced with 173 lentiviruses expressing the following sgRNAs: control, Mest distal promoter, Mest proximal promoter\#1, 
or Mest proximal promoter\#2. Seventy-two hours post-infection, cells were selected using hygromycin (1 mg/ml, Life Technologies), and clones were picked and expanded in ESC media.

\section{Generation of SAM CRISPRa ESC lines targeting Mest promoters}

E14Tg2a ESCs stably expressing the SAM system (Bonev et al. 2017) -SAM ESCs- were transfected with Lenti sgRNA(MS2)_zeo plasmids expressing the following sgRNAs: control, Mest distal promoter, Mest proximal promoter\#1, or Mest proximal promoter\#2. ESCs were selected using Zeocin $(250 \mu \mathrm{g} / \mathrm{ml}$, Life Technologies) and clones were picked and expanded.

\section{Transient transfection of SAM MEFs}

80000 MEFs stably expressing the SAM system (SAM MEFs) were transfected using Lipofectamine 2000 with 300 ng of sgRNA(MS2) plasmid expressing either one control sgRNA, one Mest distal promoter sgRNA (out of 3 different sgRNAs), one Mest proximal promoter sgRNA (out of 2 different sgRNAs), or one miR-335-putative promoter sgRNA (out of 3 different sgRNAs). Forty-eight hours later, RNAs were harvested.

\section{RNA extraction and RT-qPCR}

Total RNAs were extracted using quick-RNA miniprep kits (Zymo) and quantified on a Nanodrop. RNAs were retro-transcribed with N6 primers and M-MuLV retro-transcriptase (RT). qPCR was performed using validated primers and SYBR Green Mix (Roche) in 384-well plates on a LightCycler480 device (Roche) as described in (Varrault et al. 2018). The level of expression of each gene was normalized to the average expression levels of three housekeeping genes selected with geNorm (Vandesompele et al. 2002): Gapdh, Tbp, and Mrp/32 for ESCs and Gapdh, Tbp and Gusb for MEFs. qPCR primer sequences are listed in Supplemental Table S2.

miRNAs were retro-transcribed with gene-specific primers and multiscribe RT (Life Technologies). Their levels of expression were measured with TaqMan probes (miRNA Taqman assays \# 000546 for miR- 
335-5p, and \# 002185 for miR-335-3p). and normalized to that of U6 snoRNA (assay \# 001973)

(ThermoFisher). We found that U6 was stably expressed across samples (not shown).

\section{Visualization of RNA-seq experiment}

RNA-seq reads from mESCs -GSE75486 (Bouschet et al. 2017)- were visualized using Integrative Genomics Viewer (Robinson et al. 2011) -version: 2.8.13-.

\section{Immunofluorescence}

Immunofluorescence experiments were performed as described (Varrault et al. 2018) using antibodies directed against (species; provider; catalog number): CAS9 (mouse; Cell signalling; \#14697); NANOG (mouse; BD Pharmingen; \#560259); NESTIN (mouse; Santa Cruz; sc-33677); PAX6 (mouse; Covance; PRB-278P); POU5F1 (rabbit; Cell signalling; \#2840); TBR1 (rabbit, Cell signalling; \#49661); TUBB3 (mouse; Covance; MMS-435P). Secondary antibodies were anti-mouse or anti-rabbit coupled to Alexa Fluor ${ }^{\circledR} 488$ or Cy3 (Jackson Immunoresearch Laboratories). Nuclei were labeled with DAPI and slides were mounted with mowiol and observed under a fluorescence microscope (ImagerZ1, Zeiss). Images of organoids were obtained by tiling and stitching, and insets were taken using the apotome mode.

\section{Statistical analysis}

Statistical analysis was carried out using GraphPad Prism Version 8 (GraphPad Software, San Diego, USA). Mann-Whitney test was used for comparing differences between two groups. $p$ values $<0.05$ were considered statistically significant.

\section{COMPETING INTEREST STATEMENT}

The authors declare no competing interests.

\section{ACKNOWLEDGMENTS}

We thank Laurent Journot for helpful discussions and suggestions; Lauriane Fritsch, Boyan Bonev, and Giacomo Cavalli for SAM ESCs and SAM MEFs; Hervé Seitz, Adrien Décorsière, and Laure Garnier for 
critical reading of our draft manuscript; Chris Planque for advice; Philaé Gil and Pierre Nègre for

technical assistance; Lionel Quentin and Nicolas Boucharel for performing mycoplasma tests; members of the IGF for continuous support. pB-CAGGS-dCas9-KRAB-MeCP2 was a gift from Alejandro Chavez \& George Church (Addgene plasmid \# 110824). HyperPiggyBac Transposase (pCMV-HA-HyperpBase) was a gift of Kosuke Yusa and Allan Bradley and was kindly provided by Thomas Dibling (Sanger, Cambridge, U.K). sgRNA(MS2) cloning backbone and Lenti sgRNA(MS2)_zeo backbone were a gift 226 from Feng Zhang (Addgene plasmids \# 61424 and 61427).

\section{7}

\section{AUTHOR CONTRIBUTIONS}

T.B. conceived the project and designed the study with inputs from A.V.; I.C., M.C., and T.B. performed cell culture and qPCR experiments; A.L.D. and T.B. generated constructs; A.M. and. C.L. generated lentiviruses; C.H. generated DMH1-HCl; T.B. generated CRISPR cell lines; T.B. performed all of the analyses and drafted the manuscript; A.V. and T.B. revised the manuscript. All authors read and approved the final version of the manuscript.

\section{REFERENCES}

Bartel DP. 2018. Metazoan MicroRNAs. Cell 173: 20-51.

Baskerville S, Bartel DP. 2005. Microarray profiling of microRNAs reveals frequent coexpression with neighboring miRNAs and host genes. RNA 11: 241-247.

Bonev B, Mendelson Cohen N, Szabo Q, Fritsch L, Papadopoulos GL, Lubling Y, Xu X, Lv X, Hugnot JP, Tanay A, et al. 2017. Multiscale 3D Genome Rewiring during Mouse Neural Development. Cell 171: 557-572 e24.

Bouschet T, Dubois E, Reynes C, Kota SK, Rialle S, Maupetit-Mehouas S, Pezet M, Le Digarcher A, Nidelet S, Demolombe V, et al. 2017. In Vitro Corticogenesis from Embryonic Stem Cells Recapitulates the In Vivo Epigenetic Control of Imprinted Gene Expression. Cereb Cortex 27: 2418-2433.

Chen L, Heikkinen L, Wang C, Yang Y, Sun H, Wong G. 2019. Trends in the development of miRNA bioinformatics tools. Brief Bioinform 20: 1836-1852.

Chiang HR, Schoenfeld LW, Ruby JG, Auyeung VC, Spies N, Baek D, Johnston WK, Russ C, Luo S, Babiarz JE, et al. 2010. Mammalian microRNAs: experimental evaluation of novel and previously annotated genes. Genes Dev 24: 992-1009. 
DeVeale B, Swindlehurst-Chan J, Blelloch R. 2021. The roles of microRNAs in mouse development. Nat Rev Genet 22: 307-323.

Eiraku M, Watanabe K, Matsuo-Takasaki M, Kawada M, Yonemura S, Matsumura M, Wataya T, Nishiyama A, Muguruma K, Sasai Y. 2008. Self-organized formation of polarized cortical tissues from ESCs and its active manipulation by extrinsic signals. Cell Stem Cell 3: 519-32.

Fromm B, Billipp T, Peck LE, Johansen M, Tarver JE, King BL, Newcomb JM, Sempere LF, Flatmark K, Hovig E, et al. 2015. A Uniform System for the Annotation of Vertebrate microRNA Genes and the Evolution of the Human microRNAome. Annu Rev Genet 49: 213-242.

Gilbert LA, Larson MH, Morsut L, Liu Z, Brar GA, Torres SE, Stern-Ginossar N, Brandman O, Whitehead EH, Doudna JA, et al. 2013. CRISPR-Mediated Modular RNA-Guided Regulation of Transcription in Eukaryotes. Cell 154: 442-451.

Ha M, Kim VN. 2014. Regulation of microRNA biogenesis. Nat Rev Mol Cell Biol 15: 509-524.

He C, Li Z, Chen P, Huang H, Hurst LD, Chen J. 2012. Young intragenic miRNAs are less coexpressed with host genes than old ones: implications of miRNA-host gene coevolution. Nucleic Acids Res 40: 4002-4012.

Hinske LC, França GS, Torres HAM, Ohara DT, Lopes-Ramos CM, Heyn J, Reis LFL, Ohno-Machado L, Kreth S, Galante PAF. 2014. miRIAD—integrating microRNA inter- and intragenic data. Database 2014. https://doi.org/10.1093/database/bau099 (Accessed September 2, 2021).

Hiramuki Y, Sato T, Furuta Y, Surani MA, Sehara-Fujisawa A. 2015. Mest but Not MiR-335 Affects Skeletal Muscle Growth and Regeneration. PLOS ONE 10: e0130436.

Jones-Rhoades MW, Bartel DP, Bartel B. 2006. MicroRNAs AND THEIR REGULATORY ROLES IN PLANTS. Annu Rev Plant Biol 57: 19-53.

Kingston ER, Bartel DP. 2019. Global analyses of the dynamics of mammalian microRNA metabolism. Genome Res 29: 1777-1790.

Konermann S, Brigham MD, Trevino AE, Joung J, Abudayyeh OO, Barcena C, Hsu PD, Habib N, Gootenberg JS, Nishimasu H, et al. 2015. Genome-scale transcriptional activation by an engineered CRISPR-Cas9 complex. Nature 517: 583-8.

Labun K, Montague TG, Krause M, Torres Cleuren YN, Tjeldnes H, Valen E. 2019. CHOPCHOP v3: expanding the CRISPR web toolbox beyond genome editing. Nucleic Acids Res 47: W171W174.

Liang Y, Ridzon D, Wong L, Chen C. 2007. Characterization of microRNA expression profiles in normal human tissues. BMC Genomics 8: 1-20.

Lin Y-L, Mettling C, Portales P, Reynes J, Clot J, Corbeau P. 2002. Cell surface CCR5 density determines the postentry efficiency of R5 HIV-1 infection. Proc Natl Acad Sci 99: 15590.

Marsico A, Huska MR, Lasserre J, Hu H, Vucicevic D, Musahl A, Orom UA, Vingron M. 2013. PROmiRNA: a new miRNA promoter recognition method uncovers the complex regulation of intronic miRNAs. Genome Biol 14: 1-23. 
Meunier J, Lemoine F, Soumillon M, Liechti A, Weier M, Guschanski K, Hu H, Khaitovich P, Kaessmann H. 2013. Birth and expression evolution of mammalian microRNA genes. Genome Res 23: 34-45.

Robinson JT, Thorvaldsdóttir H, Winckler W, Guttman M, Lander ES, Getz G, Mesirov JP. 2011. Integrative genomics viewer. Nat Biotechnol 29: 24-26.

Rodriguez A, Griffiths-Jones S, Ashurst JL, Bradley A. 2004. Identification of Mammalian microRNA Host Genes and Transcription Units. Genome Res 14: 1902-1910.

Ronchetti D, Lionetti M, Mosca L, Agnelli L, Andronache A, Fabris S, Deliliers GL, Neri A. 2008. An integrative genomic approach reveals coordinated expression of intronic miR-335, miR-342, and miR-561 with deregulated host genes in multiple myeloma. BMC Med Genomics 1: 37.

Schanen BC, Li X. 2011. Transcriptional regulation of mammalian miRNA genes. Genomics 97: 1-6.

Seitz H, Royo H, Bortolin M-L, Lin S-P, Ferguson-Smith AC, Cavaillé J. 2004. A Large Imprinted microRNA Gene Cluster at the Mouse Dlk1-Gtl2 Domain. Genome Res 14: 1741-1748.

Steiman-Shimony A, Shtrikman O, Margalit H. 2018. Assessing the functional association of intronic miRNAs with their host genes. RNA 24: 991-1004.

Tomé M, López-Romero P, Albo C, Sepúlveda JC, Fernández-Gutiérrez B, Dopazo A, Bernad A, González MA. 2011. miR-335 orchestrates cell proliferation, migration and differentiation in human mesenchymal stem cells. Cell Death Differ 18: 985-995.

Vandesompele J, De Preter K, Pattyn F, Poppe B, Van Roy N, De Paepe A, Speleman F. 2002. Accurate normalization of real-time quantitative RT-PCR data by geometric averaging of multiple internal control genes. Genome Biol 3: RESEARCH0034.

Varrault A, Eckardt S, Girard B, Le Digarcher A, Sassetti I, Meusnier C, Ripoll C, Badalyan A, Bertaso F, McLaughlin KJ, et al. 2018. Mouse Parthenogenetic Embryonic Stem Cells with BiparentalLike Expression of Imprinted Genes Generate Cortical-Like Neurons That Integrate into the Injured Adult Cerebral Cortex. Stem Cells 36: 192-205.

Westholm JO, Lai EC. 2011. Mirtrons: microRNA biogenesis via splicing. Biochimie 93: 1897-1904.

Xue B, Chuang C-H, Prosser HM, Fuziwara CS, Chan C, Sahasrabudhe N, Kühn M, Wu Y, Chen J, Biton A, et al. 2021. miR-200 deficiency promotes lung cancer metastasis by activating Notch signaling in cancer-associated fibroblasts. Genes Dev 35: 1109-1122.

Yang D, Lutter D, Burtscher I, Uetzmann L, Theis FJ, Lickert H. 2014. miR-335 promotes mesendodermal lineage segregation and shapes a transcription factor gradient in the endoderm. Development 141: 514-525.

Yeo NC, Chavez A, Lance-Byrne A, Chan Y, Menn D, Milanova D, Kuo C-C, Guo X, Sharma S, Tung A, et al. 2018. An enhanced CRISPR repressor for targeted mammalian gene regulation. Nat Methods 15: 611-616. 


\author{
Zhu L, Chen L, Shi C-M, Xu G-F, Xu L-L, Zhu L-L, Guo X-R, Ni Y, Cui Y, Ji C. 2014. MiR-335, an \\ Adipogenesis-Related MicroRNA, is Involved in Adipose Tissue Inflammation. Cell Biochem \\ Biophys 68: 283-290.
}

\title{
FIGURE LEGENDS
}

Figure 1. CRISPRi on Mest suppresses the expression of hosted miR-335 in embryonic stem cells

(A) Transcription originates from the proximal promoter of Mest (P) in mouse embryonic stem cells. Integrative Genomics Viewer tracks showing coverage plot and alignment of RNA-seq reads for mouse embryonic stem cells. Reads for Mest (blue) are transcribed from the plus strand, while reads from Copg2 (pink) are transcribed from the minus strand. Chromosomal coordinates and gene annotation are from the RefSeq mm9 build. D: Mest distal promoter; P: Mest proximal promoter.

(B) Schematic of mouse Mest gene with the CRISPRi module (dCas9-KRAB-MeCP2) targeting the proximal promoter P of Mest.

(C, D) Repression of Mest promoter downregulates Mest (C) but does not affect the expression of neighboring Copg2 (D). RNAs were quantified in two CRISPRi ESC clones expressing the control sgRNA (grey) and two CRISPRi ESC clones expressing Mest sgRNA (red). Data are mean \pm sem of five independent experiments and expressed as fold change over control clone \#1. ${ }^{* *}: p<0.01$ (MannWhitney test).

(E, F) Influence of repressing Mest promoter on miR-335-3p and miR-335-5p levels. Data are mean \pm sem of five independent experiments and expressed as fold change over control clone $\# 1 .{ }^{* *}: p<0.01$ (Mann-Whitney test).

Figure 2. miR-335 expression depends on Mest promoter activity in brain organoids (A) Immunofluorescence staining on brain organoids derived from mESCs using antibodies for brain primordium markers NESTIN/PAX6 (middle panels) and TUBB3/TBR1 (right panels) after eight and 15 
days of differentiation. The top panels show entire organoids. The bottom panels are zoom-in insets of an area in organoids. Scale bars: $200 \mu \mathrm{m}$ for organoids (top panels) and $50 \mu \mathrm{m}$ for insets (bottom panels).

(B) Time course of expression of Mest and miR-335 mature products during the development of brain organoids from CRISPRi ESCs stably expressing either control sgRNA or Mest sgRNA. Heatmap shows the mean of four independent experiments performed on two CRISPRa sgRNA control and two CRISPRa sgRNA ESC clones. Heatmap was built using Morpheus. https://software.broadinstitute.org/morpheus/

Figure 3. CRISPRa on Mest increases the expression of hosted miR-335 in embryonic stem cells (A) Schematic of mouse Mest gene structure with the CRISPRa SAM - synergistic activation mediatormodule targeting the distal promoter $\mathrm{D}$ of Mest.

(B, C) Transactivation of Mest promoter upregulates Mest (B) but does not affect neighboring Copg2 expression (C). Data are mean \pm sem of five independent experiments performed on two CRISPRa sgRNA control (grey) and two CRISPRa sgRNA Mest clones (green) and expressed as fold change over control clone\#1. ${ }^{* *}: p<0.01$ (Mann-Whitney test).

(D; E) Transactivation of Mest promoter increases miR-335-3p (D) and miR-335-5p (E) levels. Data are mean \pm sem of four independent experiments and expressed as fold change over control clone\#1. $*$ : $p<0.05$ (Mann-Whitney test).

Figure 4. CRISPRa on miR-335 putative promoter does not affect miR-335 levels in MEFs

(A-C) Endogenous expression of miR-335 products and Mest is weaker in SAM MEFs than in SAM ESCs. Data are mean \pm sem of qPCR experiments performed on five MEF and five ESC samples and normalized to the average value obtained on ESCs. ${ }^{* *}: p<0.01$ (Mann-Whitney test). 
(D) Structure of mouse Mest gene with SAM targeting either the distal promoter of Mest D (D1-D2 sgRNAs), the proximal promoter of Mest P (P1-P3 sgRNAs), or the putative promoter of miR-335 ( $\mu 1$ $\mu 3$ sgRNAs).

$(\mathrm{E}-\mathrm{H})$ Levels of expression of Mest (E), Copg2 (F), miR-335-3p (G), and miR-335-5p (H) were measured after transactivation of either Mest $\mathrm{D}$ or $\mathrm{P}$ promoters or miR-335 putative promoter. SAM MEFs were transfected with plasmids expressing sgRNAs targeting Mest D (sgRNAs D1, D2, and D3), Mest $\mathrm{P}(\mathrm{P} 1$ and $\mathrm{P} 2)$, or the putative promoter of miR-335 $(\mu 1, \mu 2$, and $\mu 3)$. Data are mean \pm sem of three to four independent experiments and expressed as fold change over sgRNA control taken as $1 .{ }^{*}$ : $\mathrm{p}<0.05$ in Mann-Whitney test (comparison with sgRNA control values). None of the sgRNAs that direct the CRISPRa/SAM machinery towards the miR-335 putative promoter $(\mu 1, \mu 2$, and $\mu 3)$ altered miR335 levels.

\section{SUPPLEMENTAL FIGURE LEGENDS}

Supplemental Figure S1. Characterization of the dCas9-KRAB-MeCP2 (CRISPRi) ESC line

(A) Expression of CAS9 in parental E14Tga2 ESCs and their CRISPRi derivatives. Scale bars: $50 \mu \mathrm{m}$.

(B) Expression of pluripotency factors POU5F1 (green) and NANOG (red) in parental E14Tga2 ESCs

Supplemental Figure S2. Efficient CRISPRi of Mest when targeting its proximal promoter

(A) Structure of mouse Mest gene with the CRISPRi module (dCa9-KRAB-MeCP2) directed to either the distal $(D)$ (sgRNA D, green) or proximal (P) (sgRNAs P1 -purple- and P2 -red-) promoter of Mest. The sgRNA control (grey) has no match in the mouse genome. sgRNA control, sgRNA D, sgRNA P1, or sgRNA P2. RNAs were measured by RT-qPCR. Data are 
390 mean \pm sem of seven independent experiments and expressed as fold change over control sgRNA. *: $391 \mathrm{p}<0.05,{ }^{* * *}: \mathrm{p}<0.001$ in Mann-Whitney test (comparison with sgRNA control values). Only the sgRNAs 392 targeting the proximal promoter repressed Mest.

393 Supplemental Figure S3. Efficient CRISPRa of Mest when targeting its distal promoter

394 (A) Structure of mouse Mest gene with the CRISPRa tool SAM targeting either the distal (sgRNA D, 395 green) or the proximal (sgRNAs P1 -purple- and P2 -red-) promoter of Mest. The sgRNA control (grey) 396 has no match in the mouse genome.

397 (B, C) Transactivation of Mest distal promoter upregulates Mest expression (B) and does not affect 398 neighboring Copg2 expression (C). CRISPRa SAM ESCs were transduced with lentivirus expressing 399 either SgRNA control, D, P1, or P2. Mest (B) and Copg2 (C) were measured by RT-qPCR. Data are 400 mean \pm sem of four independent experiments. * $p<0.1$ in Mann-Whitney test. Only the sgRNA targeting 401 the distal promoter upregulated Mest RNA.

402 Supplemental Table S1. Sequences of sgRNAs

403 Supplemental Table S2. Primers used for qPCR assays 
bioRxiv preprint doi: https://doi.org/10.1101/2021.09.15.458166; this version posted September 15, 2021. The copyright holder for this preprint (which was not certified by peer review) is the author/funder, who has granted bioRxiv a license to display the preprint in perpetuity. It is made available under aCC-BY-NC-ND 4.0 International license.

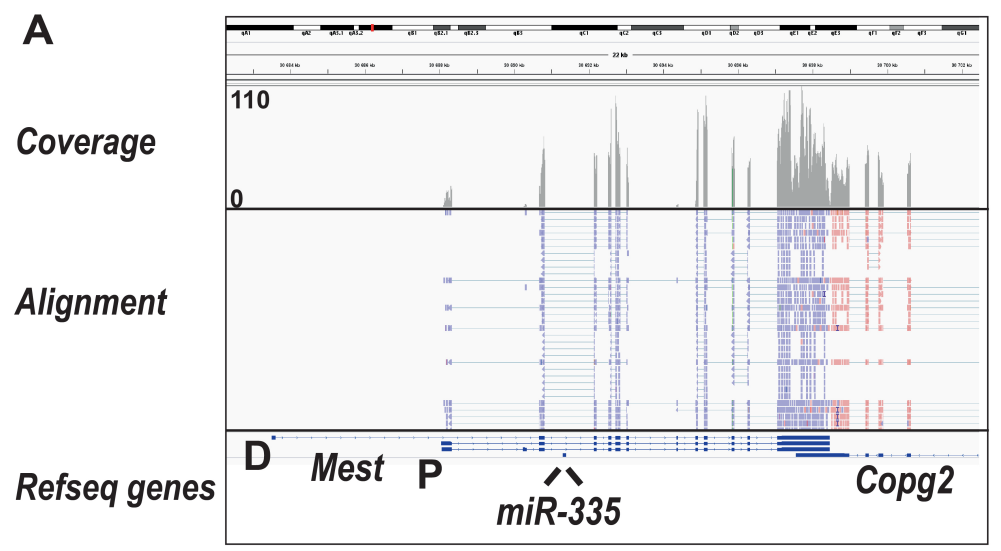

Courtes_Fig. 1

B

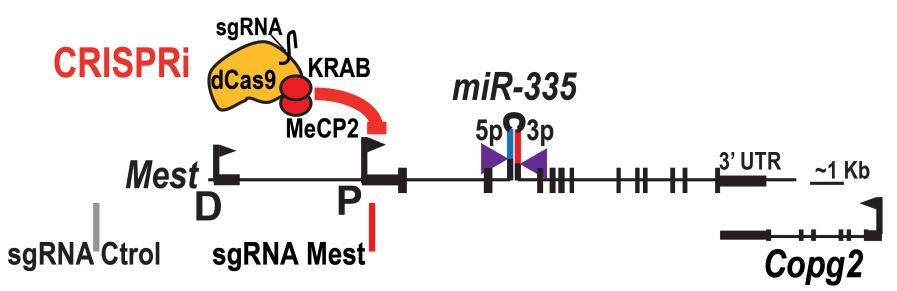

C

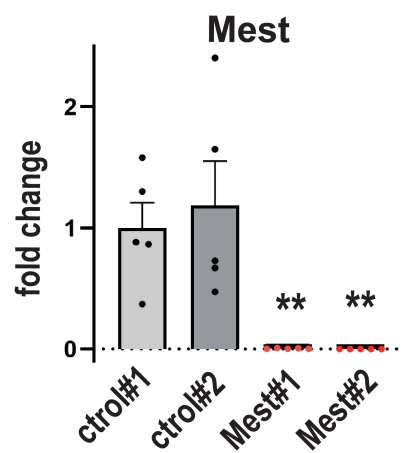

$\mathbf{E}$

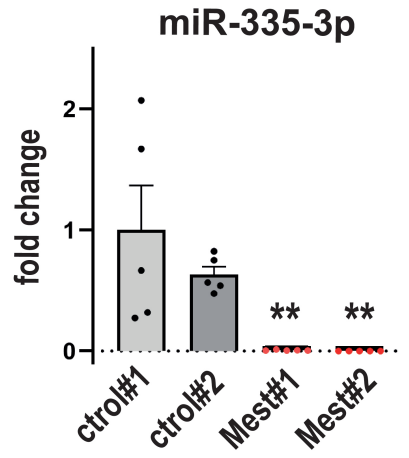

D

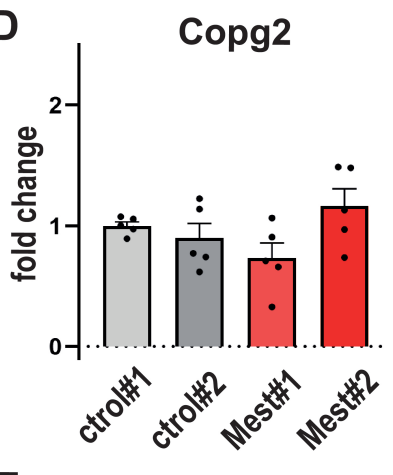

$\mathbf{F}$

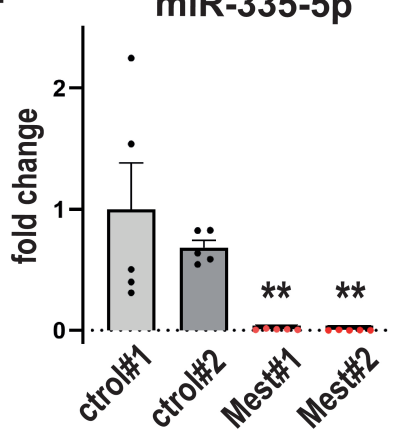

Figure 1. CRISPRi on Mest suppresses the expression of hosted miR-335 in embryonic stem cells

(A) Transcription originates from the proximal promoter of $M e s t(P)$ in mouse embryonic stem cells. Integrative Genomics Viewer tracks showing coverage plot and alignment of RNA-seq reads for mouse embryonic stem cells. Reads for Mest (blue) are transcribed from the plus strand, while reads from Copg2 (pink) are transcribed from the minus strand. Chromosomal coordinates and gene annotation are from the RefSeq $\mathrm{mm} 9$ build. D: Mest distal promoter; P: Mest proximal promoter.

(B) Schematic of mouse Mest gene with the CRISPRi module (dCas9-KRAB-MeCP2) targeting the proximal promoter of Mest.

(C, D) Repression of Mest promoter downregulates Mest (C) but does not affect the expression of neighboring Copg2 (D). RNAs were quantified in two CRISPRi ESC clones expressing the control sgRNA (grey) and two CRISPRi ESC clones expressing Mest sgRNA (red). Data are mean \pm sem of five independent experiments and expressed as fold change over control clone \#1. ${ }^{* *}: p<0.01$ (Mann-Whitney test).

$(E, F)$ Influence of repressing Mest promoter activity on miR-335-3p and miR-335-5p levels. Data are mean \pm sem of five independent experiments and expressed as fold change over control clone $\# 1$. ${ }^{* *}:$ p $<0.01$ (Mann-Whitney test). 


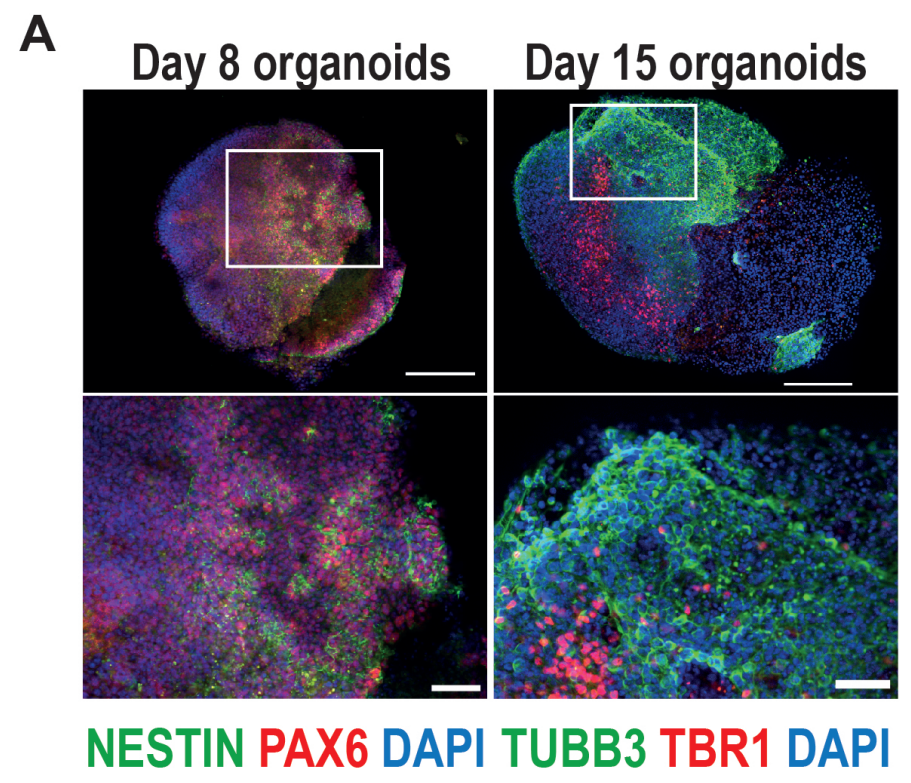

Courtes_Fig. 2

B

row min

row max

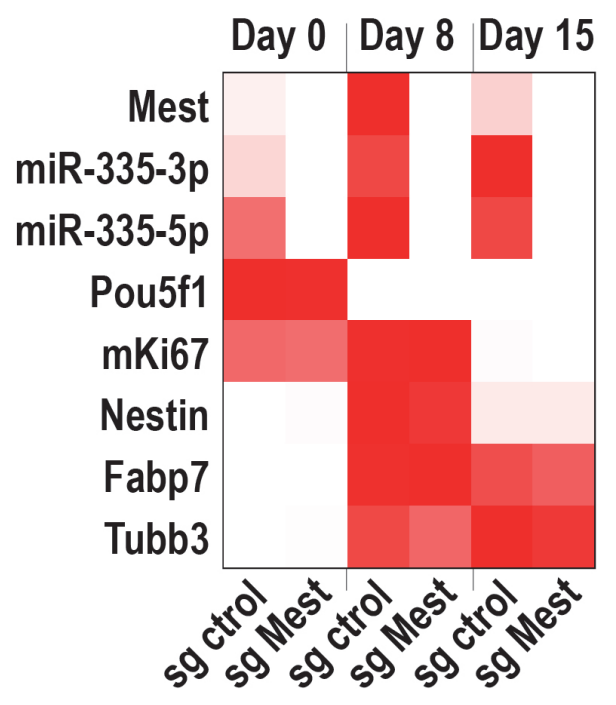

Figure 2. miR-335 expression depends on Mest promoter activity in brain organoids

(A) Immunofluorescence staining on brain organoids derived from mESCs using antibodies for brain primordium markers NESTIN/PAX6 (middle panels) and TUBB3/TBR1 (right panels) after eight and 15 days of differentiation. The top panels show entire organoids. The bottom panels are zoom-in insets of an area in organoids. Scale bars: $200 \mu \mathrm{m}$ for organoids (top panels) and $50 \mu \mathrm{m}$ for insets (bottom panels).

(B) Time course of expression of Mest and miR-335 mature products during brain organogenesis from CRISPRi ESCs stably expressing either control sgRNA or Mest sgRNA. Heatmap shows the mean of four independent experiments performed on two CRISPRa sgRNA control and two CRISPRa sgRNA ESC clones. Heatmap was built using Morpheus. https://software.broadinstitute.org/morpheus/ 


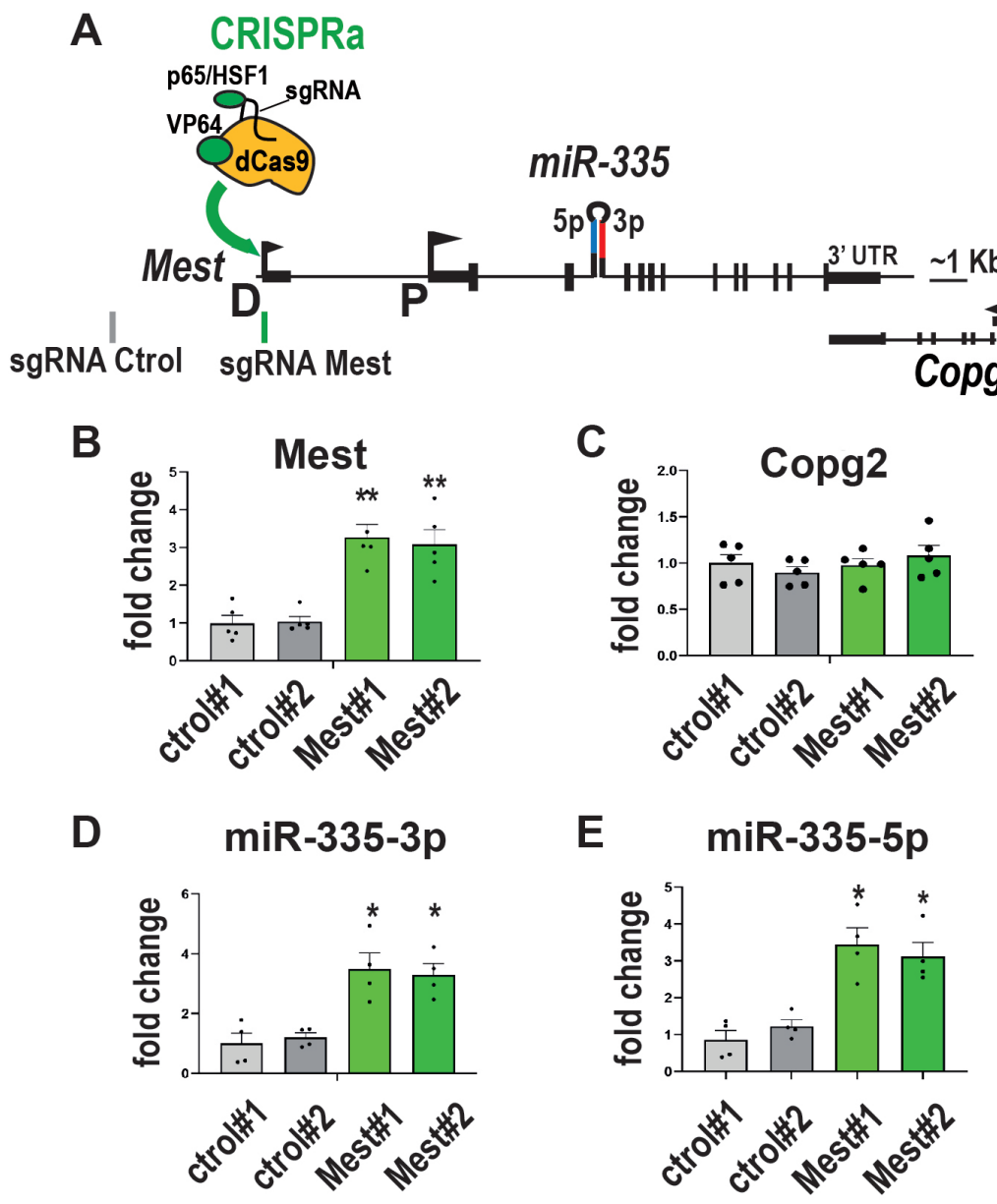

Courtes_Fig. 3

Figure 3. CRISPRa on Mest increases the expression of hosted miR-335 in embryonic stem cells

(A) Schematic of mouse Mest gene structure with the CRISPRa SAM - synergistic activation mediator- module targeting the distal promoter D of Mest.

(B, C) Transactivation of Mest promoter upregulates Mest (B) but does not affect neighboring Copg2 expression (C). Data are mean \pm sem of five independent experiments performed on two CRISPRa sgRNA control (grey) and two CRISPRa sgRNA Mest clones (green) and expressed as fold change over control clone\#1.**:p<0.01 (Mann-Whitney test).

(D; E) Transactivation of Mest promoter increases miR-335-3p (D) and miR-335-5p (E) levels. Data are mean \pm sem of four independent experiments and expressed as fold change over control clone\#1. *:p<0.05 (Mann-Whitney test). 

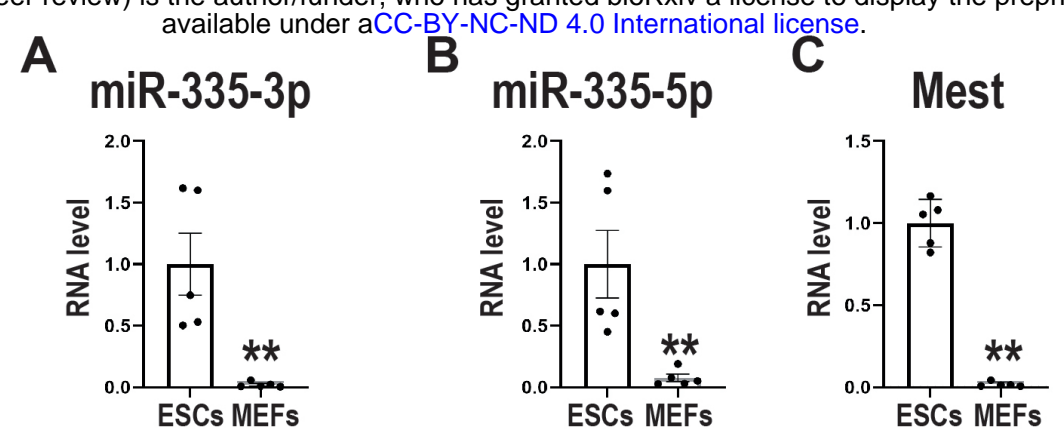

Courtes_Fig. 4
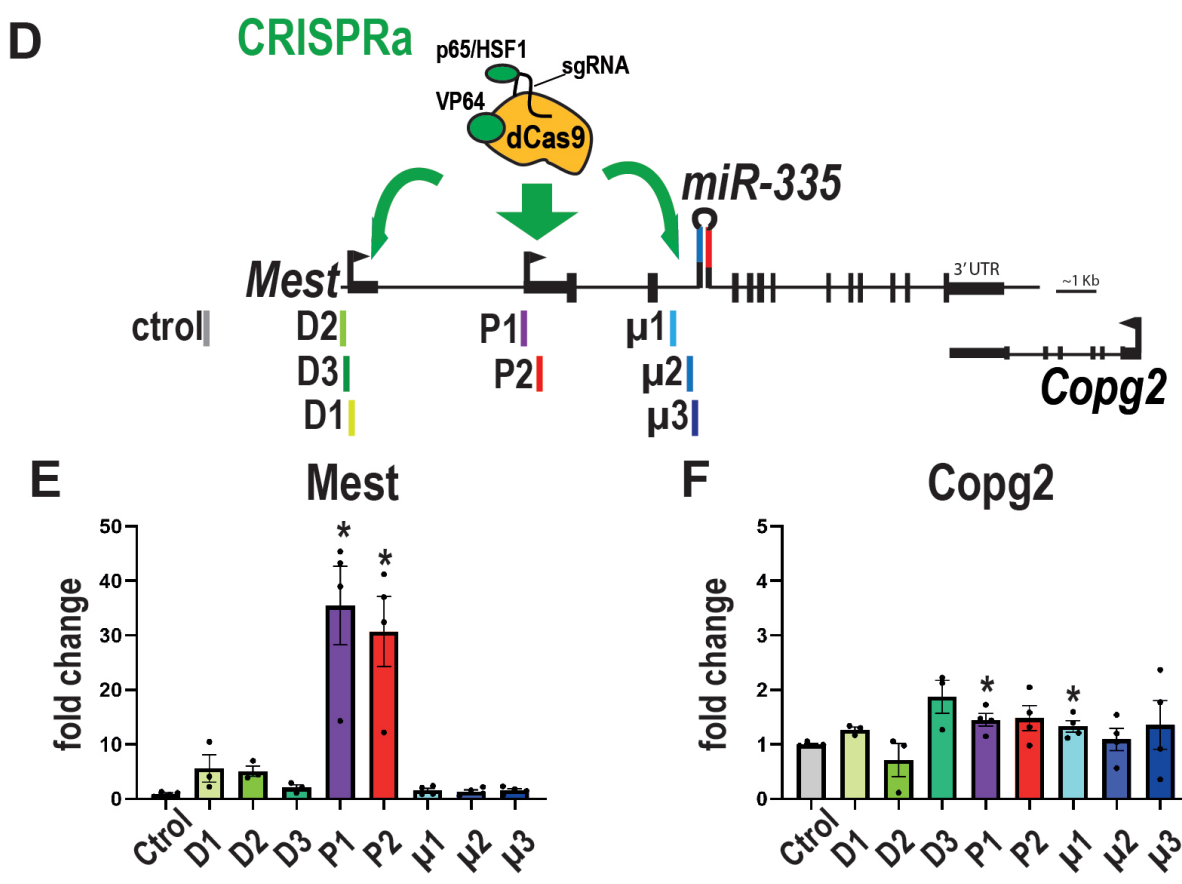

$F$

Copg2
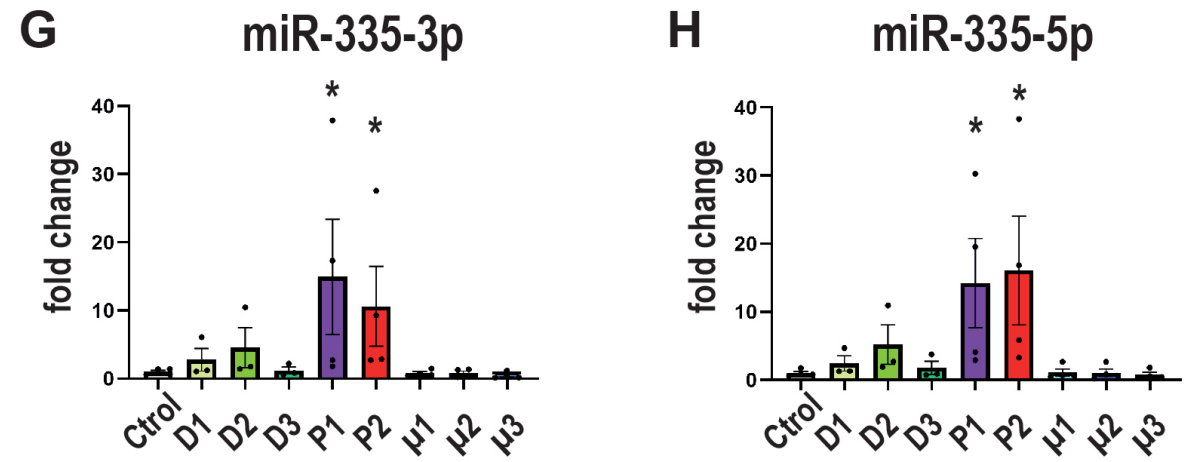

Figure 4. CRISPRa on miR-335 putative promoter does not affect miR-335 levels in MEFs

(A-C) Endogenous expression of miR-335 products and Mest is weaker in SAM MEFs than in SAM ESCs. Data are mean \pm sem of qPCR experiments performed on five MEF and five ESC samples and normalized to the average value obtained on ESCs. ${ }^{* *}: p<0.01$ (Mann-Whitney test).

(D) Structure of mouse Mest gene with SAM targeting either the distal promoter of Mest D (D1-D2 sgRNAs), the proximal promoter of Mest P (P1-P3 sgRNAs), or the putative promoter of miR-335 ( $\mu 1-\mu 3$ sgRNAs).

(E-H) Levels of expression of Mest (E), Copg2 (F), miR-335-3p (G), and miR-335-5p (H) were measured after transactivation of either Mest $\mathrm{D}$ or $\mathrm{P}$ promoters or miR-335 putative promoter. SAM MEFs were transfected with plasmids expressing sgRNAs targeting Mest D (sgRNAs D1, D2, and D3), Mest P (P1 and P2), or the putative promoter of miR-335 $(\mu 1, \mu 2$, and $\mu 3)$. Data are mean \pm sem of three to four independent experiments and expressed as fold change over sgRNA control taken as 1. *: $p<0.05$ in Mann-Whitney test (comparison with sgRNA control values). Note that none of the sgRNAs that direct the CRISPRa/SAM machinery towards the miR-335 putative promoter $(\mu 1, \mu 2$, and $\mu 3)$ altered miR-335 levels. 


\section{A}

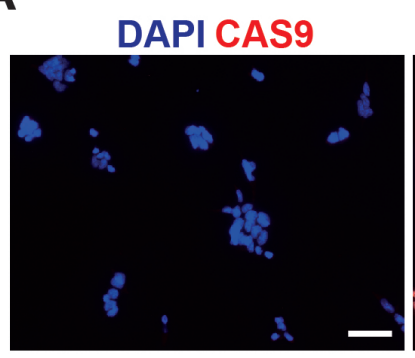

Parental ESCs
DAPICAS9

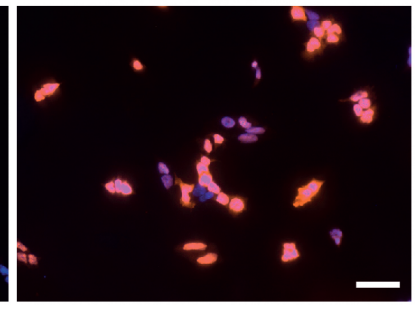

CRISPRi ESCs

Courtes_Suppl. Fig. S1

B
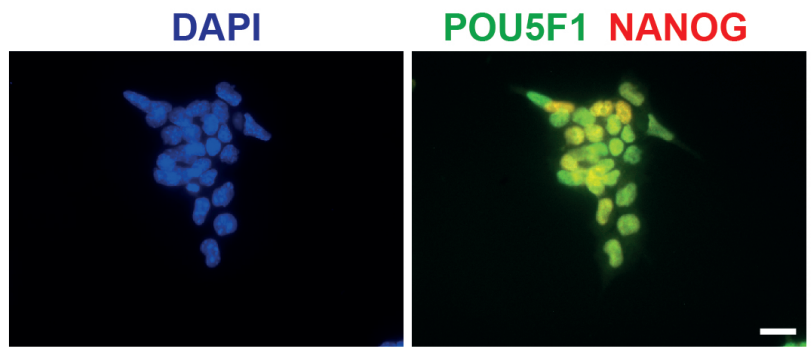

Parental ESCs
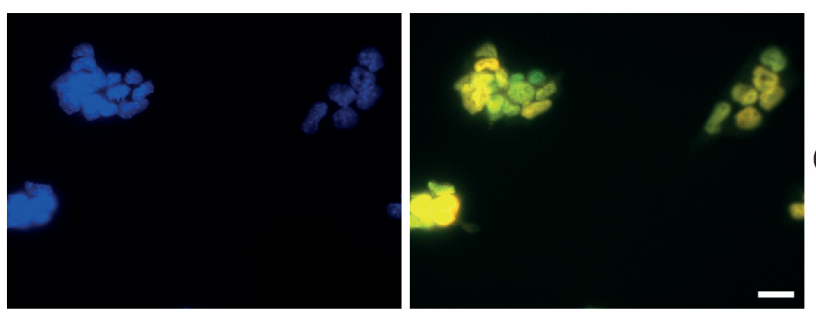

CRISPRi ESCs

Supplemental Figure S1. Characterization of the dCas9-KRAB-MeCP2 (CRISPRi) ESC line

(A) Expression of CAS9 in parental E14Tga2 ESCs and their CRISPRi derivatives. Scale bars: 50 $\mu \mathrm{m}$.

(B) Expression of pluripotency factors POU5F1 (green) and NANOG (red) in parental E14Tga2 ESCs and their CRISPRi derivatives. Scale bars: $20 \mu \mathrm{m}$. 


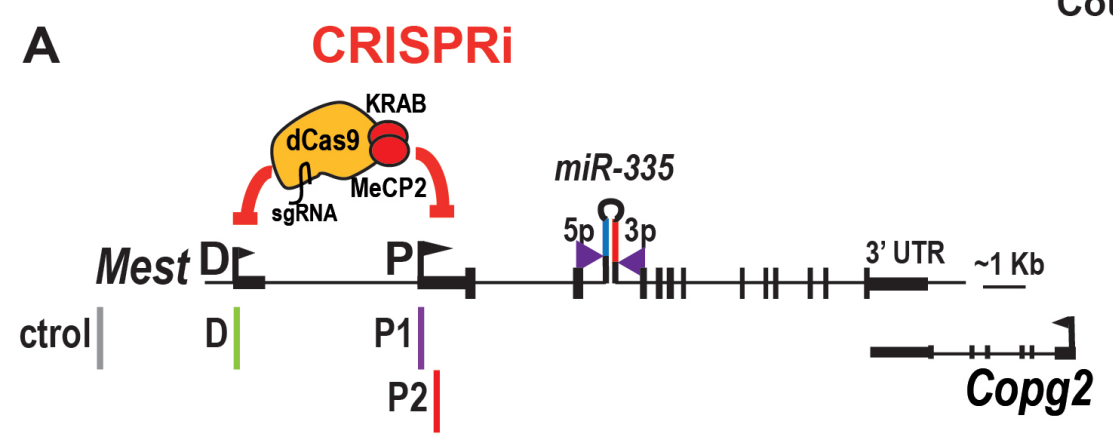

B

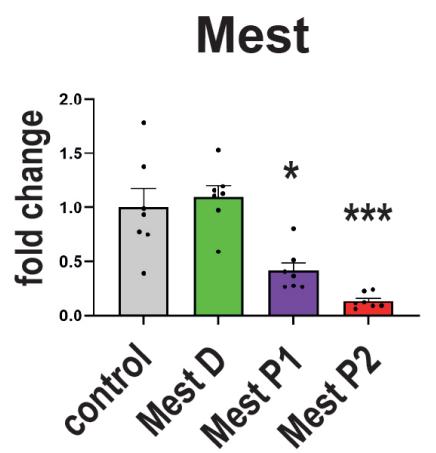

Courtes_Suppl. Fig. S2

C

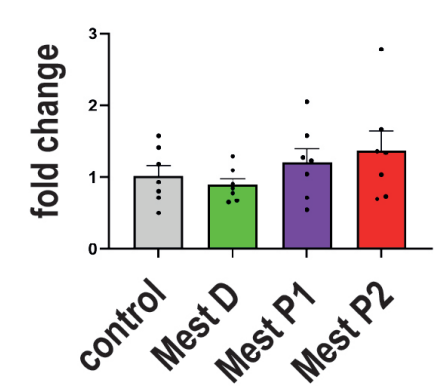

Supplemental Figure S2. Efficient CRISPRi of Mest when targeting its proximal promoter

(A) Structure of mouse Mest gene with the CRISPRi module (dCa9-KRAB-MeCP2) directed to either the distal $(D)$ (sgRNA D, green) or proximal (P) (sgRNAs P1 -purple- and P2 -red-) promoter of Mest. The sgRNA control (grey) has no match in the mouse genome.

(B, C) Repression of Mest proximal promoter downregulates Mest expression (B) and does not affect neighboring Copg2 expression (C). CRISPRi ESCs were transduced with lentiviruses expressing either sgRNA control, sgRNA D, sgRNA P1, or sgRNA P2. RNAs were measured by RT-qPCR. Data are mean \pm sem of seven independent experiments and expressed as fold change over control sgRNA. *: $p<0.05,{ }^{* * *}: p<0.001$ in Mann-Whitney test (comparison with sgRNA control values). Only the sgRNAs targeting the proximal promoter repressed Mest. 


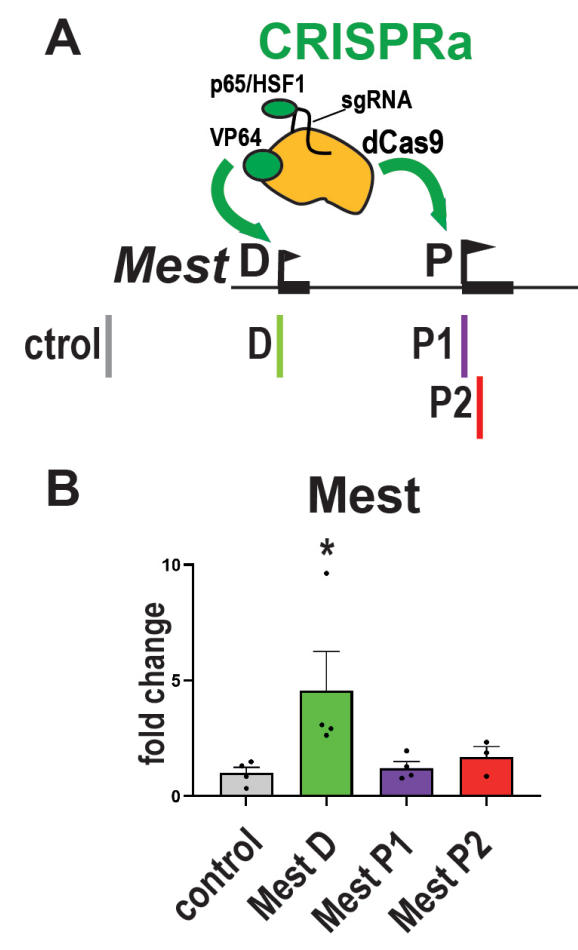

Courtes_Suppl. Fig. S3

C Copg2

miR-335
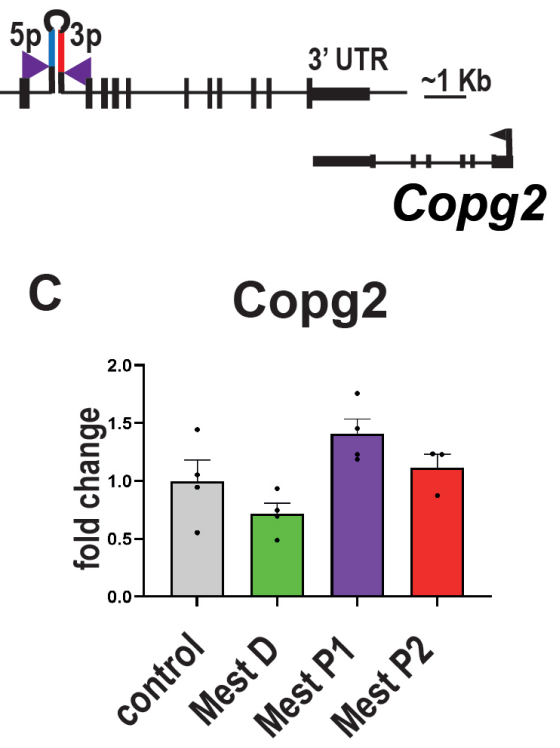

Supplemental Figure S3. Efficient CRISPRa of Mest when targeting its distal promoter

(A) Structure of mouse Mest gene with the CRISPRa tool SAM targeting either the distal (sgRNA D, green) or the proximal (sgRNAs P1 -purple- and P2 -red-) promoter of Mest. The sgRNA control (grey) has no match in the mouse genome.

(B, C) Transactivation of Mest distal promoter upregulates Mest expression (B) and does not affect neighboring Copg2 expression (C). CRISPRa SAM ESCs were transduced with lentivirus expressing either SgRNA control, D, P1, or P2. Mest (B) and Copg2 (C) were measured by RTqPCR. Data are mean \pm sem of four independent experiments. *: $p<0.1$ in Mann-Whitney test. Only the sgRNA targeting the distal promoter upregulated Mest RNA. 


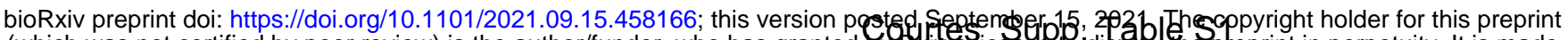

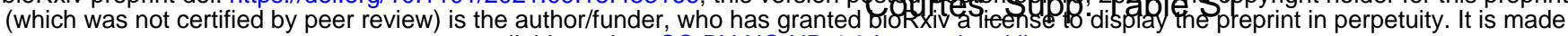
available under aCC-BY-NC-ND 4.0 International license.

sgRNA

Control (Addgene \#61424) GGGTCTTCGAGAAGACCTGT

Control (Addgene \#61427)

Mest $\mathrm{p} 1$

Mest p2

Mest d1

Mest d2

Mest d3

miR-335 $\mu 1$

$\operatorname{miR}-335 \mu 2$

$\operatorname{miR}-335 \mu 3$ sequence $5^{\prime}$ to $3^{\prime}$

GGAGACGGGATACCGTCTCT

GCTCAGTGGGCTTTAAAAGT

GGCGCAGCAGCTTTCCTCTG

GAGGGCCCAGCGGGGCGGCG

AACCAGGGGAAGGACAGCTG

CAACCCAAATCACCTGCCCC

TTTTGAGCGCCCCTAGTGTC

TTACAACAGCATTTGGAGAT

GAAGAAACCGAGAAACAGAT

Supplemental Table S1. Sequence of sgRNAs 


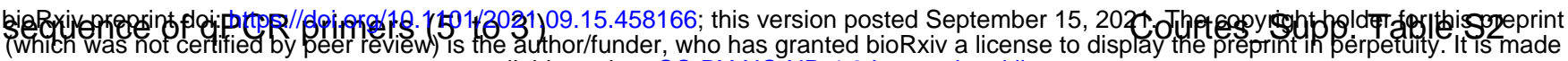

\begin{tabular}{ccl} 
Symbol & Gene ID & \multicolumn{1}{c}{ Forward Seq. } \\
Mest & 17294 & CAACAATGACGGCAACCTGGT \\
Copg2 & 54160 & TGATGTGGTTAAACGATGGATAAATGAAG \\
Tubb3 & 22152 & CCAGTGCGGCAACCAGATAGG \\
Nanog & 71950 & GCCTCTCCTCGCCCTTCCTCT \\
Pou5f1 & 18999 & CTGTAGGGAGGGCTTCGGCACTT \\
Fabp7 & 12140 & TCCAGCTGGGAGAAGAGTT \\
Nes & 18008 & CGGAGAGGGAGCAGCACCAA \\
Gapdh & 14433 & GGAGCGAGACCCCACTAACA \\
Gusb & 110006 & GATTCAGATATCCGAGGGAAAGG \\
Mki67 & 17345 & TCCAGACTTCCACAGAGAC \\
Mrpl32 & 75398 & AGGTGCTGGGAGCTGCTACA \\
Tbp & 21374 & ACTTCGTGCAAGAAATGCTGAAT
\end{tabular}

Reverse Seq.

TCTGAATTTCTTCCTTTGATTAATGTACTGTA

TGGAGACAGCAAGCCGATCAT

AAAGGCGCCAGACCGAACACT

CCACCGCTTGCACTTCATCCTT

CTGAGGGCCAGGCAGGAGCACGAG

CCAACCGAACCACAGACTTA

GGCCTCCCCCACAGCATCCT

ACATACTCAGCACCGGCCTC

GCCAACGGAGCAGGTTGA

TTCACCTTCATCCAGATTCAC

AAAGCGACTCCAGCTCTGCT

CAGTTGTCCGTGGCTCTCTTATT

Supplemental Table S2. Primers used for qPCR assays 